\title{
Thermal diffusion forced Rayleigh scattering setup optimized for aqueous mixtures
}

\author{
Simone Wiegand, ${ }^{1, *}$ Hui Ning, ${ }^{1, \dagger}$ and Hartmut Kriegs ${ }^{1, \ddagger}$ \\ ${ }^{1}$ Forschungszentrum Jülich GmbH, \\ IFF - Weiche Materie, D-52428 Jülich, Germany
}

(Dated: October 19, 2007)

\begin{abstract}
We developed a thermal diffusion forced Rayleigh scattering (TDFRS) set-up operating at a writing wavelength of $980 \mathrm{~nm}$, which corresponds to an absorption band of water with an absorption coefficient of approximately $0.5 \mathrm{~cm}^{-1}$. Therefore, aqueous mixtures require no dye to convert the light into heat energy. Especially for aqueous system with a complex phase behavior such as surfactant systems the addition of a water soluble dye can cause artifacts. The infrared-TDFRS (IR-TDFRS) set-up has been validated for water/ethanol mixtures with water weight fractions between $c=0.5-0.95$ and in a temperature range between $T=15^{\circ} \mathrm{C}$ to $T=35^{\circ} \mathrm{C}$. Comparison with literature data shows an excellent agreement. The addition of a small amount $\left(c_{d y e} \sim 10^{-6}\right.$ wt) adsorbing dye at the writing wavelength allows also the investigation of organic mixtures. We investigated the three binary mixtures of dodecane, isobutylbenzene and 1,2,3,4 tetrahydronaphthalene at a weight fraction of $c=0.5$ at a temperature of $25^{\circ} \mathrm{C}$ and found good agreement with the Soret coefficients, which had been obtained in a benchmark test under the same conditions. Therefore the presented set-up is suitable for the investigation of the thermal diffusion behavior in aqueous and organic mixtures and in the case of aqueous systems the addition of a dye can be avoided.
\end{abstract}

PACS numbers: 


\section{INTRODUCTION}

Thermal diffusion describes the migration of molecules in a temperature gradient. The molecular origin of the effect, also called Ludwig-Soret effect, is one of the unsolved problems in physical chemistry. In some cases even qualitative predictions are impossible. The main practical applications are separation processes $^{1,2}$ such as thermal field flow fractionation of polymers and colloids or isotope separation, characterization of geochemical processes ${ }^{3,4}$ and combustion $^{5}$.

There are many experimental methods such as thermal diffusion cells ${ }^{6-8}$, thermogravitational columns ${ }^{9}$, thermal lens method ${ }^{10,11}$, holographic grating methods ${ }^{12-14}$, field-flow fractionation ${ }^{15}$ and microscopic methods ${ }^{16,17}$ to investigate the thermal diffusion behavior of simple and complex liquid mixtures. Except for the thermal lens method, the field-flow fractionation and the microscopic technique all methods have been validated in a benchmark test ${ }^{18}$ or show consistent results for organic ${ }^{7,19,20}$ and aqueous mixtures ${ }^{7,21-24}$. Besides the reliability of the methods sometimes certain aspects come to the fore. For instance lies the strength of the thermogravitational columns in the possibility to investigate multi component systems, while the other methods are limited to binary mixtures unless special systems ${ }^{22}$ are studied or modified set-ups are used ${ }^{25}$. Another important criteria is the equilibration time, which can be in the order of 10 hours and more for slow diffusing systems such as polymers and colloids. In this respect optical methods, such as transient grating methods often denoted as thermal diffusion forced Rayleigh scattering (TDFRS), thermal lens and a micron scale diffusion cells ${ }^{8}$ are superior to other methods.

The TDFRS methods allows the reliable investigation of simple liquids ${ }^{26}$, polymeric solutions $^{27}$ and colloidal suspensions ${ }^{28}$, but since the early works by Thyagarajan and Lallemand ${ }^{29}$ and $\mathrm{Pohl}^{30}$ the investigated mixtures have always been slightly colored with some dye to create a sufficient absorption between $0.2 \mathrm{~cm}^{-1}$ and $1 \mathrm{~cm}^{-1}$. Ideally, the dye is inert, which means that there is no photobleaching and no dye contribution to the diffraction signal. For organic mixtures, it has been shown that the addition of an organic dye results in very small dye contribution to the signal (on the order of $0.5 \%$ ). These contributions do not influence the mean values of the transport coefficients but lead to slightly asymmetric error bars $^{31}$. In the case of aqueous systems it is more difficult to find an inert dye. Typically the spectroscopic properties of dyes depend on $\mathrm{pH}$, ionic strength and other parameters. In 

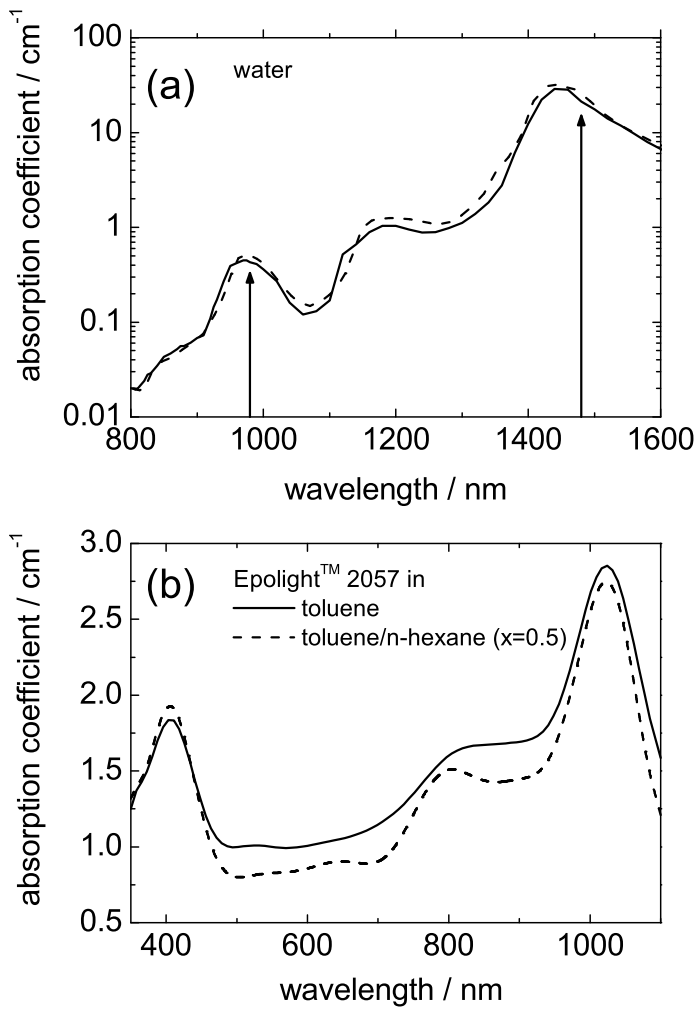

FIG. 1: (a) Absorption spectrum of water determined by Hale and Querry ${ }^{33}$ (solid line) and Palmer and Williams ${ }^{34}$ (dashed line). The arrows correspond to a wavelength of $\lambda=980 \mathrm{~nm}$ and $\lambda=1480 \mathrm{~nm}$. The shorter wavelength is used in the thermal lens set-up by the group of Piazza ${ }^{11}$ and in the IR-TDFRS in this work, while the fluorescence microscopy method in the group of Braun operates at the longer wavelength ${ }^{17}$. (b) Absorption spectrum of Epolight ${ }^{T M} 2057$ in toluene (solid line) and in a toluene/ $n$-hexane mixture (dashed line) with a toluene mole fraction of $x=0.5$.

recent studies on aqueous surfactant systems ${ }^{14,32}$ it was found that the dye has a physical effect on the micelles of some non-ionic surfactant systems and leads to a second slow thermophoretic mode. Therefore, it would be desirable to have a set-up which works especially for aqueous systems without the addition of dye.

In the literature there are two set-ups among the optical methods, which use the laser light for heating, which investigate especially aqueous systems without the addition of dye. One is the thermal lens set-up in the group of Piazza ${ }^{11}$, which works with a diode laser at $\lambda=980 \mathrm{~nm}$ and the other is a fluorescence microscopy method developed in the group of 
Braun $^{17}$, which uses a solid state laser with a center wavelength of $\lambda=1480 \mathrm{~nm}$. According to the absorption spectrum of water (c.f. Fig. 1(a)) the absorption for the two methods in pure water is $0.5 \mathrm{~cm}^{-1}$ and $21 \mathrm{~cm}^{-1}$, respectively. In principle it should be possible to build a TDFRS set-up using one of the near infrared wavelength as writing beam for the investigation of aqueous solutions.

The goal of this paper is to present the Infrared-TDFRS (IR-TDFRS) set-up, which operates at a writing beam wavelength of $\lambda=980 \mathrm{~nm}$. We will discuss, why we have chosen as a first approach the shorter wavelength and which modifications had to be made in comparison to TDFRS-set-up in the visible wavelength range. Although there are no benchmark values available for aqueous system we found reliable data for the system water/ethanol in the literature ${ }^{7,21-24}$. We compare our data with those literature data. Additionally we also investigated the three binary mixtures of dodecane, isobutylbenzene and 1,2,3,4 tetrahydronaphthalene at a weight fraction or $c=0.5$ at a temperature of $25^{\circ} \mathrm{C}$ by adding a small amount of inert dye which has a strong absorption band at $\lambda=980 \mathrm{~nm}$ (c.f. Fig. 1(b)). Those three organic mixtures have been investigated by four different groups in a benchmark ${ }^{18}$.

\section{EXPERIMENT}

\section{A. Sample Preparation.}

The solvents ethanol (BASF, absolute), dodecane (C12H26) (Sigma-Aldrich, 99+\%), isobutylbenzene (IBB) (Fluka, $\geq 99 \%$ ) and 1,2,3,4 tetrahydronaphthalene (THN) (SigmaAldrich, 99+\%) were used without further purification. We took deionized water (Milli-Q). The organic mixtures were prepared as follows: First a very small amount (roughly $10^{-6} \mathrm{wt}$ ) of the dye Epolight ${ }^{T M} 2057$ (Epolin), was dissolved in the solvents. For each solution the optical density was adjusted to $0.2-0.3 \mathrm{~cm}^{-1}$ at a wavelength of $\lambda=980 \mathrm{~nm}$. The aqueous mixtures did not contain any dye. The mixtures were prepared as follows: Samples for the IR-TDFRS measurements were prepared just before the measurement to avoid evaporation. The solutions were directly filtered into the sample cells (Nylon, $0.2 \mu \mathrm{m}$ ). The temperature was controlled by a circulating water bath with an uncertainty of $\Delta T=0.02 \mathrm{~K}$. 


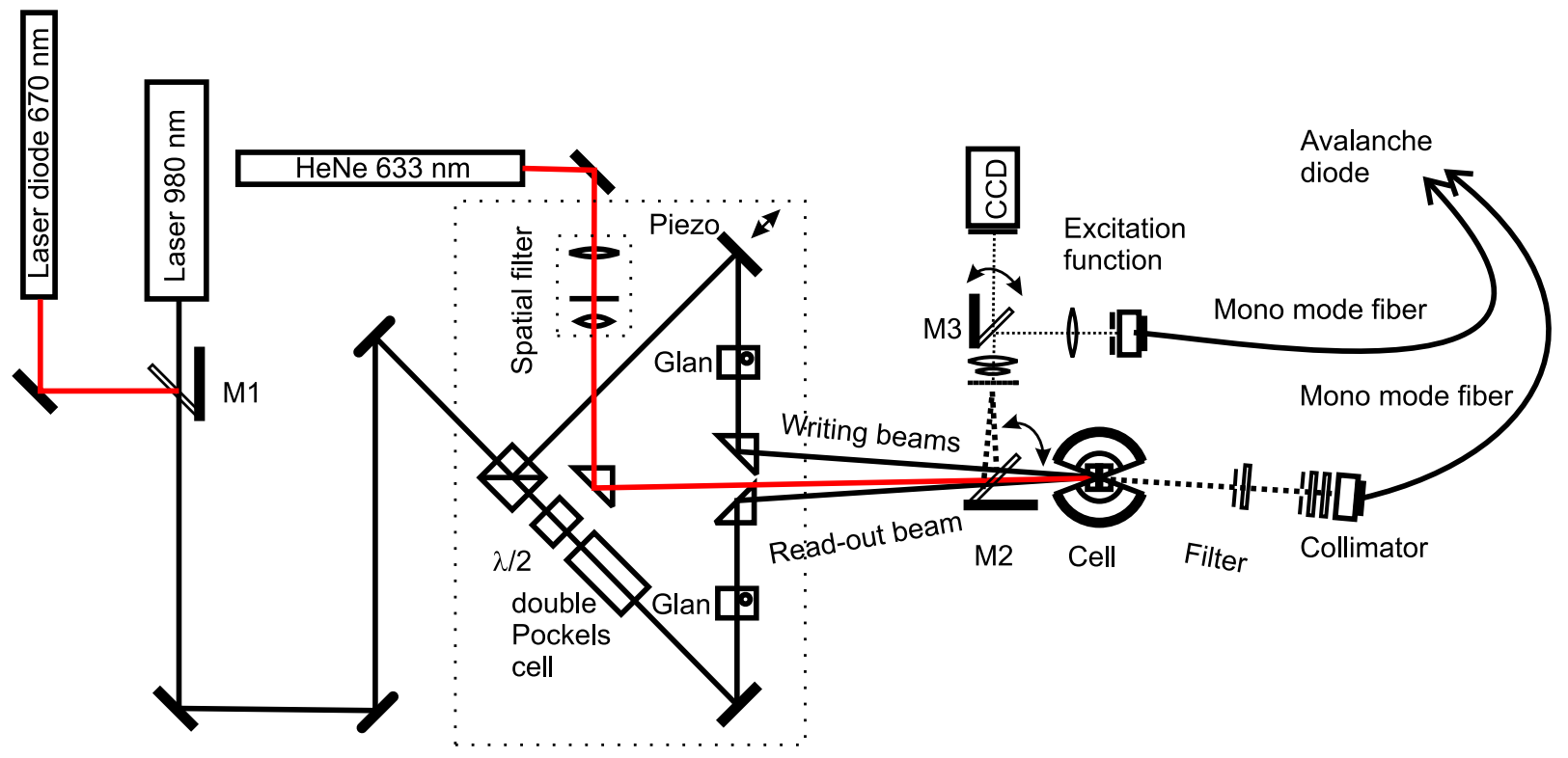

FIG. 2: Sketch of the IR-TDFRS set-up

\section{B. Refractive index increment measurements.}

The temperature derivatives of the refractive index $(\partial n / \partial T)_{p, c}$ at a constant pressure and concentration were determined in the temperature range $T \pm 3{ }^{\circ} \mathrm{C}$ using a Michelson interferometer ${ }^{35}$. The values are listed in Table I. The determined values (c.f. Tab I) agree typically better than $1 \%$ with the data determined by Kolodner ${ }^{23}$.

Refractive index increments with concentration $(\partial n / \partial c)_{p, T}$ at a constant pressure and temperature were measured using an Abbe refractometer. The data are compared with data by Kolodner ${ }^{23}$ and are listed in Table I. In the experimental investigated range, which has been studied in both works, the data compare well with the literature data.

The numerical values of both refractive index increments $(\partial n / \partial T)_{p, c}$ and $(\partial n / \partial c)_{p, T}$ are listed in Table I

\section{TDFRS experiment and sample cell}

The experimental set-up of TDFRS is sketched in Fig. 2. The interference grating is written in by a grating-stabilized diode laser with a tapered semiconductor amplifier (TA100, Toptica) with a maximum output power of 1 Watt operating at a wavelength of $980 \mathrm{~nm}$. To allow for an easier alignment we use a visible laser diode operating at $670 \mathrm{~nm}$ with a 
TABLE I: Water weight fraction $c$, Refractive index increments with respect to temperature $(\partial n / \partial T)_{p, C}$ and concentration $(\partial n / \partial c)_{p, T}$ of water/ethanol mixtures, Soret coefficient $S_{\mathrm{T}}$, thermal diffusion coefficient $D_{\mathrm{T}}$ and diffusion coefficient $D$.

\begin{tabular}{|c|c|c|c|c|c|c|}
\hline$c$ & $\begin{array}{l}\mathrm{T} / \\
{ }^{\circ} \mathrm{C}\end{array}$ & $\begin{array}{c}(\partial n / \partial T)_{p, c} / \\
10^{-4} \mathrm{~K}^{-1}\end{array}$ & $\begin{array}{c}(\partial n / \partial c)_{p, T} / \\
10^{-2}\end{array}$ & $\begin{array}{c}S_{\mathrm{T}} / \\
10^{-3} \mathrm{~K}^{-1}\end{array}$ & $\begin{array}{c}D_{\mathrm{T}} / \\
10^{-8} \mathrm{~cm}^{2} \mathrm{~s}^{-1} \mathrm{~K}^{-1}\end{array}$ & $\begin{array}{c}D / \\
10^{-6} \mathrm{~cm}^{2} \mathrm{~s}^{-1}\end{array}$ \\
\hline 0.50 & 15 & -3.20 & -2.66 & 5.30 & 1.34 & 2.53 \\
\hline 0.60 & 15 & -2.87 & -4.07 & 3.68 & 1.10 & 2.97 \\
\hline 0.80 & 15 & -1.55 & -7.79 & -3.66 & -1.87 & 5.11 \\
\hline 0.90 & 15 & -0.92 & -7.41 & -7.78 & -5.57 & 7.15 \\
\hline 0.95 & 15 & -0.75 & -6.58 & -8.14 & -6.49 & 7.97 \\
\hline 0.50 & 25 & -3.31 & -2.53 & 4.33 & 1.67 & 3.86 \\
\hline 0.60 & 25 & -3.00 & -3.54 & 3.36 & 1.43 & 4.27 \\
\hline 0.80 & 25 & -1.84 & -6.65 & -3.33 & -2.09 & 6.29 \\
\hline 0.90 & 25 & -1.26 & -6.91 & -6.32 & -5.99 & 9.48 \\
\hline 0.95 & 25 & -1.10 & -6.45 & -7.08 & -7.89 & 11.15 \\
\hline 0.50 & 35 & -3.41 & -2.10 & 3.91 & 2.36 & 6.04 \\
\hline 0.60 & 35 & -3.10 & -3.36 & 2.78 & 1.63 & 5.87 \\
\hline 0.80 & 35 & -2.09 & -6.30 & -2.63 & -2.40 & 9.13 \\
\hline 0.90 & 35 & -1.57 & -6.47 & -5.57 & -6.98 & 12.55 \\
\hline 0.95 & 35 & -1.40 & -6.28 & -6.10 & -8.46 & 13.88 \\
\hline 0.50 & 45 & -3.52 & -1.92 & 3.19 & 2.90 & 9.10 \\
\hline 0.60 & 45 & -3.22 & -2.97 & 2.49 & 2.37 & 9.51 \\
\hline 0.80 & 45 & -2.31 & -5.60 & -2.41 & -2.63 & 10.91 \\
\hline 0.90 & 45 & -1.83 & -6.12 & -4.75 & -7.64 & 16.08 \\
\hline 0.95 & 45 & -1.66 & -6.17 & -5.05 & -8.08 & 15.99 \\
\hline
\end{tabular}


maximum output power below $1 \mathrm{~mW}$. By using the flip-mirror $M 1$ a preliminary alignment can be done in the visible. Special care has to be taken for some optical elements. For instance the reflectivity of the beam splitter strongly depends on the wavelength.

In the infrared the beam splitter divides the beam into two beams of approximately equal intensity. One of the beams is reflected by a mirror mounted on a piezo attenuator, which is used for phase shift and stabilization to obtain the heterodyne signal. The polarization direction of the other beam is rotated $90^{\circ}$ by a half wave plate. The double Pockels cell (EM508/2, Leysop) rotates the polarization by $\pm 90^{\circ}$, so that the polarization direction of this beam is parallel or anti parallel to the original polarization. This leads to a shift of the grating by $180^{\circ}$, which means that a dark fringe (cold) becomes bright (warm) and vice versa. To achieve a better contrast the beams are polarized by two Glan Thompson. The longer double Pockels cells is required to achieve the necessary polarization rotation for writing beam at a wavelength of $\lambda=980 \mathrm{~nm}$, which is roughly a factor two larger than the wavelength of the writing beam in the visible set-up ${ }^{14}$. If we would have chosen the longer wavelength at $\lambda=1480 \mathrm{~nm}$, we would have needed two Pockels cells in series to achieve the required rotation of the polarization direction.

The grating is read by a He-Ne laser (124, Spectra Physics) with a output intensity of $22 \mathrm{~mW}$ at $\lambda=632.8 \mathrm{~nm}$. We use a spatial filter to improve the beam quality and to focus the beam on the mono mode fiber in the detection arm. In contrast to the set-up in the visible the read out wave length is longer than the writing wavelength, the read-out beam has to be between the two writing beams in order to fulfill the Bragg condition (see Sec. IID). This small angle of the writing beams can causes some problems due to the high scattering intensity at low angles. The intensity of the diffracted beam is measured by an avalanche diode, which has a higher quantum efficiency than the photomultiplier used before. Additionally it has the advantage that it is still sensitive in the near infrared, so that the excitation function and the diffracted beam can be recorded with the same detector.

The flip mirror $M 2$ in front of the cell was used to image the diffraction grating on a CCD camera to determine the grating vector. A typical grating vector in the experiments is approximately $q \sim 3200 \mathrm{~cm}^{-1}$ which corresponds to a fringe spacing around $d=20 \mu \mathrm{m}$. The grating vector is comparable with the one we use in the visible set-up. A third flip mirror M3 in front of the CCD camera is used to record the excitation function. For the measurement of the excitation function the same monomode fiber without collimator and 
the same recording procedure is used.

\section{Operating conditions}

The steady state amplitude of the temperature grating $T_{\mathrm{A}}$ is given by ${ }^{24}$

$$
T_{\mathrm{A}}=\frac{\alpha I_{w} d^{2}}{4 \pi^{2} \kappa}
$$

with the absorptions coefficient $\alpha$, the intensity of the writing beam $I_{w}$, the fringe spacing $d$ and the heat conductivity $\kappa$. Assuming a fringe spacing of $d=20 \mu \mathrm{m}$ and a $I_{w}=0.1 \mathrm{~W} \mathrm{~cm}^{-2}$ this results approximately in a temperature amplitude of $T_{\mathrm{A}} \sim 80 \mu \mathrm{K}$ in pure water. This is a slightly larger temperature difference than in the visible TDFRS setup, but certainly will change if other mixtures are investigated. All results are always extrapolated to zero intensity so that convection effects can be excluded. The thermal diffusivity of pure water at $T=23^{\circ} \mathrm{C}$ has been determined to $D_{\mathrm{th}}=(1.47 \pm 0.02) \times 10^{-3} \mathrm{~cm}^{2} / \mathrm{s}$, which agrees within the error bars with the literature value of $D_{\mathrm{th}}=(1.40 \pm 0.07) \times 10^{-3} \mathrm{~cm}^{2} / \mathrm{s}^{36}$.

The read-out beam needs to fulfill the Bragg condition, which implies that the magnitude of the scattering vectors for the writing $q_{w}$ and the reading $q_{r}$ beam have to be equal. The diffraction efficiency depends on the sample thickness $s$, the incident angle of the read-out beam $\theta_{r}$, the wavelength $\lambda$. the amplitude of the spatial refractive index modulation $\Delta n$ and the misalignment of the Bragg condition as explained in Eq.78 in Ref. ${ }^{37}$. The diffraction efficiency at the Bragg angle $\theta_{B}$ is given by

$$
\eta=\sin ^{2}\left(\frac{\pi \cdot \Delta n \cdot s}{\lambda \cos \theta_{B}}\right)
$$

which implies that in first approximation for small angles the diffraction efficiency is independent of the Bragg angle. A typical grating vector of $q \sim 3200 \mathrm{~cm}^{-1}$ corresponds to a scattering angle $\theta_{w} \sim 2.9^{\circ}$ for the writing beams, which leads to an angle $\theta_{r} \sim 0.9^{\circ}$ between the optical axis and the readout beam. For our thin sample cell thickness of $s=0.2 \mathrm{~mm}$ the Bragg condition is not critical. An error in the order of $1^{\circ}$ reduces the efficiency only by $10 \%$. 


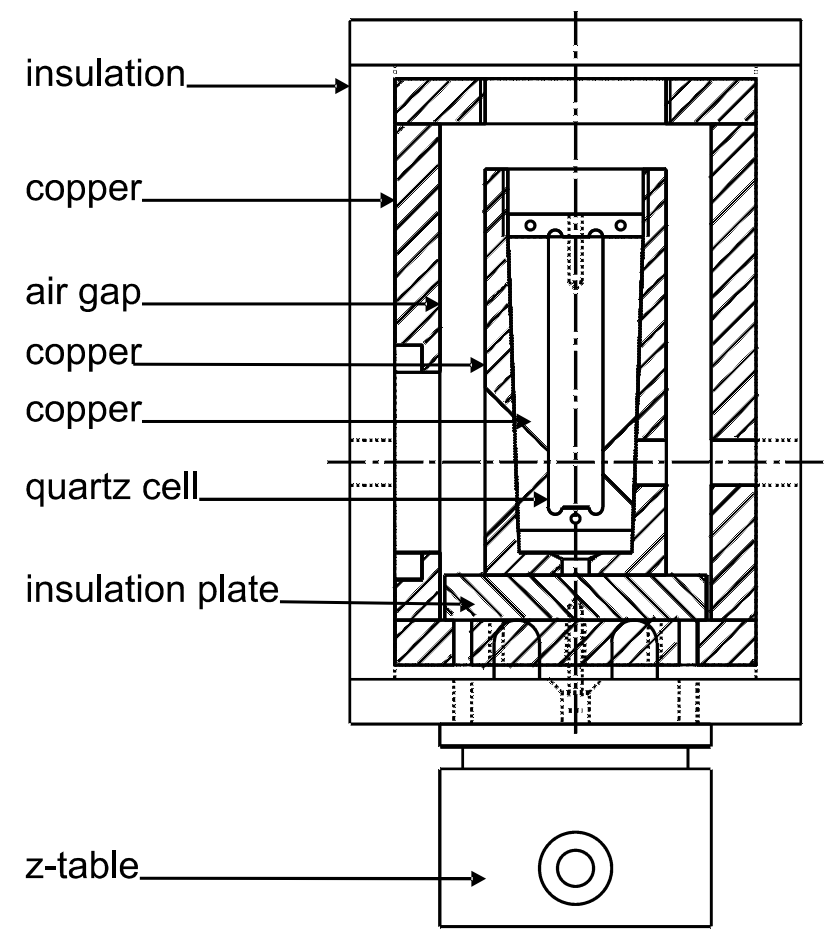

FIG. 3: Sketch of the thermostated sample holder.

\section{E. Sample cell}

The thermostated sample holder is sketched in Fig. 3. The quartz sample with a thickness of $s=0.2 \mathrm{~mm}$ is placed in two half shells, which slide conically in the inner copper cylinder of the sample holder. This inner part can be heated electrically and is thermally insulated by an air gap and a polyacetal plate at the bottom. Through the outer part flows thermostated water on a helical path through the cell. On the side of the incoming beam (left side in Fig. 3) a copper piece in the outer thermostated part can be removed during the alignment. The cell is completely insulated by a cover made of polyacetal. The entire cell can be moved by a $z$-table up and down to look for a good position of the sample cell to optimize the signal to noise ratio. Due to the small angle of the read-out beam the background scattering is often very high. It also turned out that during temperature changes the scattering intensities varied, which might due to some interference effects by the sample cell windows.

The TDFRS measurements were carried out in a temperature range from 15.0 to $45.0^{\circ} \mathrm{C}$. 


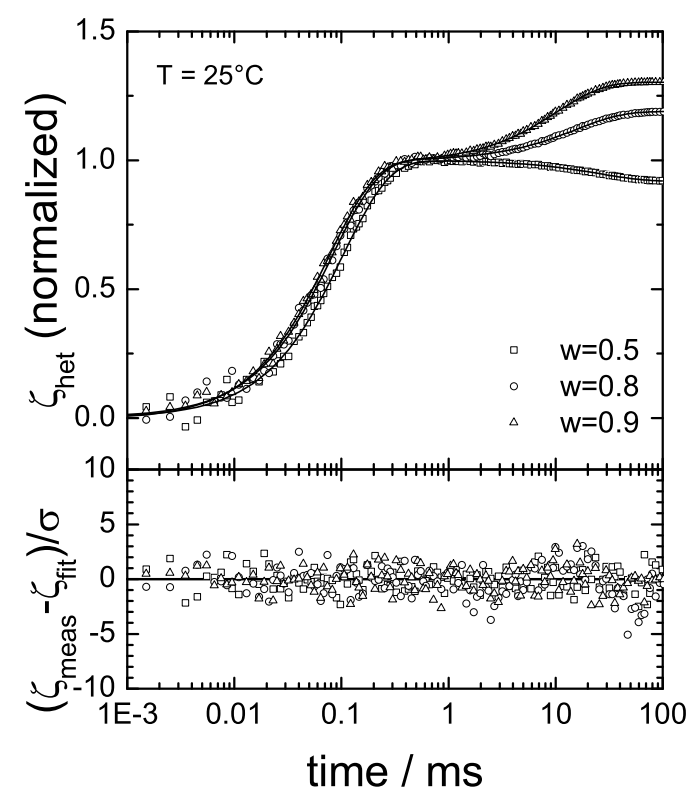

FIG. 4: Typical heterodyne diffraction signals normalized to the thermal plateau plotted as function of time for three different water/ethanol mixtures. The shown curves correspond to three different water weight fractions $c=0.5,0.8$ and 0.9 at $25^{\circ}$. The solid lines are fits to the data according to Eq. 4. The bottom part of the figure shows the residuals normalized to the standard deviation.

\section{F. Data analysis}

The heterodyne signal intensity of the read-out laser is proportional to the amplitude of the refractive index difference $\Delta n(T, c)$ as

$$
\Delta n(T, c)=\left(\frac{\partial n}{\partial T}\right)_{p, c} \Delta T+\left(\frac{\partial n}{\partial c}\right)_{p, T} \Delta c .
$$

where $\Delta T$ and $\Delta c$ are the difference in temperature and concentration, respectively.

The total intensity $\zeta_{\text {het }}(t)$ normalized to the thermal signal is related to the Soret coefficient as

$$
\zeta_{\text {het }}(t)=1-\left(\frac{\partial n}{\partial T}\right)_{p, c}^{-1}\left(\frac{\partial n}{\partial c}\right)_{p, T} S_{\mathrm{T}} c(1-c)\left(1-e^{-q^{2} D t}\right) .
$$

where $q$ is the length of the grating vector and $D$ is the mutual diffusion coefficient.

To determine the transport coefficients, Eq. 4 is fitted to the measured heterodyne signal 


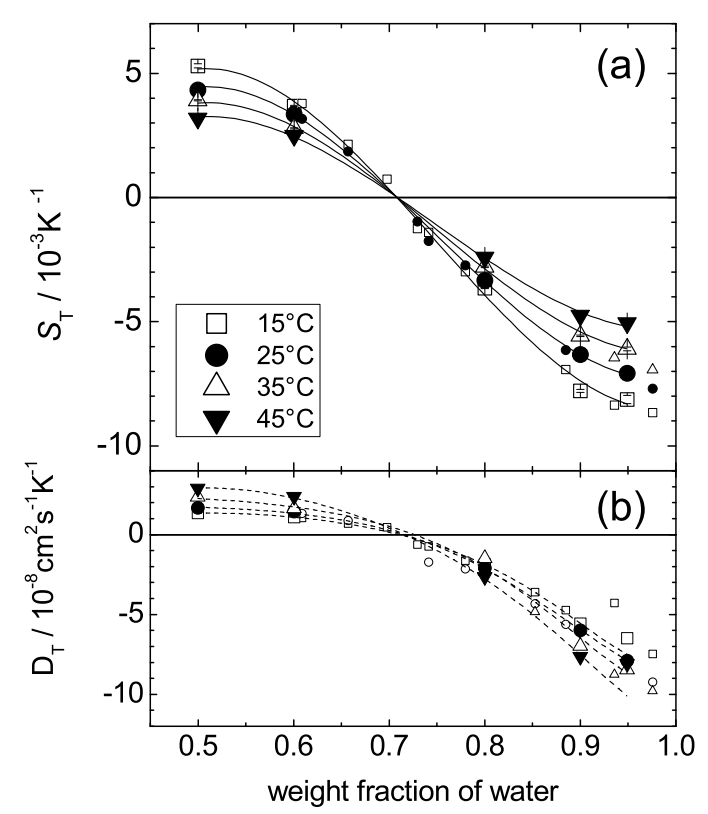

FIG. 5: (a) Soret coefficient $S_{\mathrm{T}}$ of water/ethanol as a function of mass fraction of water $c$ at $15^{\circ} \mathrm{C}$ $(\square), 25^{\circ} \mathrm{C}(\bullet), 35^{\circ} \mathrm{C}(\triangle)$ and $45^{\circ} \mathrm{C}(\boldsymbol{\nabla})$ measured with the IR-TDFRS. The smaller symbols refer to data points by Kolodner et al. ${ }^{23}$ and the lines are fits according to Eq. 5. (b) The thermal diffusion coefficients $D_{\mathrm{T}}$ in the same temperature and concentration range. Identical symbols have been used and the dashed lines are guides to the eye.

(Fig. 4) using contrast factors $(\partial n / \partial c)_{p, T}$ and $(\partial n / \partial T)_{p, c}$ which are measured separately. The residuals are typical in the $3 \sigma$ range and show no systematic deviations (Fig. 4).

\section{RESULTS}

\section{A. Water/ethanol}

The best characterized aqueous mixture in the literature is the system water/ethanol. The system has been studied by four different groups with three different methods: thermal diffusion cells ${ }^{7,23}$, thermogravitational cell $^{21}$ and TDFRS $^{22}$.

We investigated the system at five concentrations in the range between $c=0.5-0.95$ and four different temperatures between $T=15-45^{\circ} \mathrm{C}$. The obtained Soret and thermal diffusion coefficients are displayed in Fig.5. The smaller symbols of the same shape refer to 


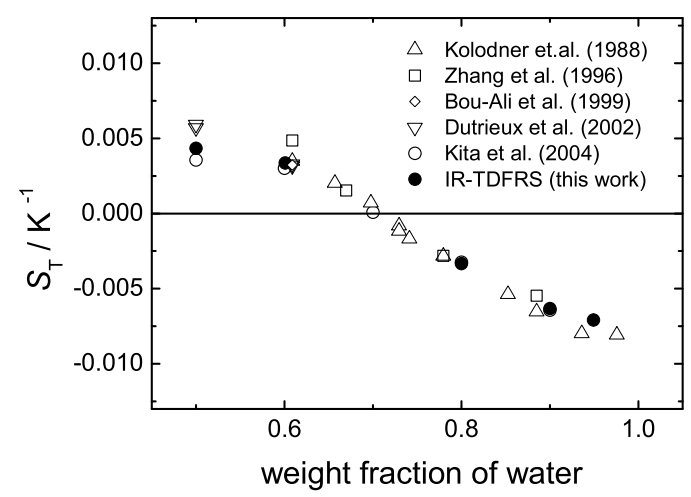

FIG. 6: Soret coefficient $S_{\mathrm{T}}$, of water/ethanol as a function of mass fraction of water $c$ by Kolodner et al. ${ }^{23}$ at $20^{\circ} \mathrm{C}(\triangle)$, Zhang et al. ${ }^{7}$ at $25^{\circ} \mathrm{C}(\square)$, Bou-Ali et al. ${ }^{38}$, Dutrieux et al. ${ }^{21}$ at $22.5^{\circ} \mathrm{C}(\nabla)$, Kita et al. at $23^{\circ} \mathrm{C}^{22}(\bigcirc)$ and this work at $25^{\circ}$

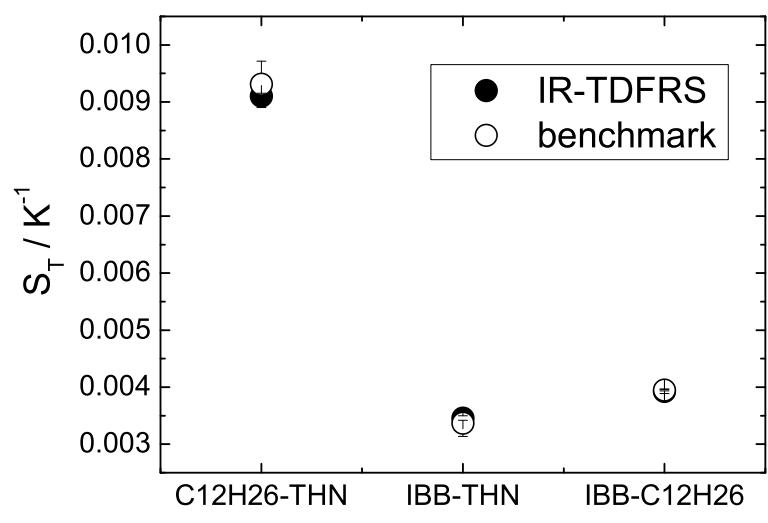

FIG. 7: Soret coefficient $S_{\mathrm{T}}$, of the three binary benchmark mixtures of dodecane (C12H26), isobutylbenzene (IBB) and 1,2,3,4 tetrahydronaphthalene (THN) at a mass fraction of $c=0.5$ at $T=25^{\circ} \mathrm{C}$ in comparison with the benchmark values ${ }^{18}$.

literature data obtained by Kolodner ${ }^{23}$ at the same temperature. In general we find good agreement typically better than $5 \%$ for all temperatures.

As recently, proposed by Wittko and Köhler ${ }^{39}$ the concentration and temperature dependence of the Soret coefficient might be described by the following empirical formula.

$$
S_{\mathrm{T}}(c, T)=\alpha(c) \beta(T)+S_{\mathrm{T}}^{i}
$$


with

$$
\begin{array}{r}
\alpha(c)=a_{0}+a_{1} c+a_{2} c^{2}+a_{3} c^{3} \\
\beta(T)=1+b_{1}\left(T-T_{0}\right)+b_{2}\left(T-T_{0}\right)^{2} .
\end{array}
$$

$T_{0}=25^{\circ} \mathrm{C}$ is an arbitrary reference temperature. The solid lines in Fig. 5 correspond to a description of the data by Eq. 5 with $a_{0}=(-0.065 \pm 0.007) \mathrm{K}^{-1}, a_{1}=(0.330 \pm$ $0.028) \mathrm{K}^{-1}, a_{2}=(-0.492 \pm 0.040) \mathrm{K}^{-1}, a_{3}=(0.220 \pm 0.018) \mathrm{K}^{-1}, b_{1}=(-0.016 \pm 0.001)$ $\mathrm{K}^{-1}, b_{2}=(0.0001 \pm 0.0001) \mathrm{K}^{-2}$ and $S_{\mathrm{T}}^{i}=0.0 \pm 0.0003 \mathrm{~K}^{-1}$. Within the experimental uncertainty $S_{\mathrm{T}}^{i}=0$, which means that for ethanol/water the concentration $c_{w}^{ \pm}$at which the Soret coefficient changes its sign does not depend on temperature and is equal to the concentration $c_{w}^{\times}$where the Soret coefficient isotherms intersect. The same observation has also been made for acetone/water and DMSO/water ${ }^{40}$. In those aqueous mixture the structural change in the liquid mixture is in the investigated temperature range independent of the temperature. On the other hand this structural change is strongly correlated with the sign change of the thermal diffusion motion. Both observations are responsible for the fact that the isotherms intersect at $c_{w}^{ \pm}$with $S_{\mathrm{T}}=0$.

In Fig. 6 we compare the Soret coefficients for water/ethanol determined with the IRTDFRS with all other literature data ${ }^{7,21-23,38}$ obtained at ambient temperatures. Typically the agreement is within $5 \%$, but for the low water content higher deviations with some literature data can be observed. At $c=0.6$ and $c=0.6$ deviations in the order of $30 \%$ can be observed compared to Zhang et al and Dutrieux et al, respecticely.

\section{B. Organic mixtures}

Additionally we investigated a well characterized system toluene/n-hexane at $23^{\circ} \mathrm{C}$ and the three binary benchmark mixtures of dodecane, isobutylbenzene and 1,2,3,4 tetrahydronaphthalene at a weight fraction of $c=0.5$ at a temperature of $25^{\circ} \mathrm{C}$. For the organic systems we need to add a small amount of dye to increase the absorption at the writing wavelength. The determined Soret coefficients $S_{\mathrm{T}}=9.10 \pm 0.05 \times 10^{-3} \mathrm{~K}^{-1}, S_{\mathrm{T}}=3.46 \pm 0.04 \times 10^{-3} \mathrm{~K}^{-1}$ and $S_{\mathrm{T}}=3.93 \pm 0.04 \times 10^{-3} \mathrm{~K}^{-1}$ for dodecane/1,2,3,4 tetrahydronaphthalene, isobutylbenzene/1,2,3,4 tetrahydronaphthalene and isobutylbenzene/dodecane, respectively are displayed in Figure 7. For all systems we find fairly good agreement. The best agreement was 
found for the system isobutylbenzene/dodecane with the largest contrast, which also had in the benchmark test the smallest error bar.

For other organic mixtures such has toluene/n-hexane we found a dependence on the dye content, but for a dye content in the order of $10^{-6} \mathrm{wt}$ which corresponds to an absorption of $0.25 \mathrm{~cm}^{-1}$ the obtained values agree for three different weight fractions $c=0.264,0.517$ and 0.762 at $23^{\circ} \mathrm{C}$ with the literature data ${ }^{19}$.

\section{DISCUSSION AND CONCLUSION}

In the present work we can conclude that the IR-TDFRS set-up is optimal for aqueous systems because no dye is required, but with the addition of an infrared absorbing dye it is also possible to investigate organic mixtures. For both classes of systems we obtained reliable data which compare well with the literature.

In the present configuration it was easier to realize the setup with the shorter infrared writing wavelength $\lambda=980 \mathrm{~nm}$. Although the longer writing wavelength $\lambda=1480 \mathrm{~nm}$ has a 40-times higher absorbance and therefore a laser with much less power would be needed, other complications show up. Choosing a writing wavelength of $\lambda=1480 \mathrm{~nm}$ would require two double Pockels cells to reach the necessary polarization rotation. This would cause an even stronger asymmetry in the two separated beams, which can cause coherence problems.

With the realized set-up it will be possible to investigate the previously studied surfactant systems without the addition of dye. Preliminary experiments showed already that the second mode disappears. Therefore we will be able to add systematically charged cosurfactants to study the charge effect for this system class.

\section{Acknowledgments}

We are very grateful for the excellent support from the mechanic workshop in the IFF of the research center in Jülich. We would also like to thank Alfons Becker for the help with the technical drawings. We grateful for fruitful discussions with Malte Kleemeier and Rio Kita. This work was partially supported by the Deutsche Forschungsgemeinschaft grant Wi 
1684.

* s.wiegand@fz-juelich.de; http://www.fz-juelich.de/iff/personen/S.Wiegand/

$\dagger$ h.ning@fz-juelich.de; http://www.fz-juelich.de/iff/personen/H.Ning/

$\ddagger$ h.kriegs@fz-juelich.de; http://www.fz-juelich.de/iff/personen/H.Kriegs/

1 M. E. Schimpf and J. C. Giddings, Macromolecules 20, 1561 (1987).

2 K. Clusius and G. Dickel, Naturwissenschaften 27, 148 (1939).

3 H. C. Helgeson, Pure \& Appl. Chem. 57, 31 (1885).

4 P. Costeseque, D. Fargue, and P. Jamet, in Thermal nonequilibrium phenomena in fluid mixtures, edited by W. Köhler and S. Wiegand (Springer, Berlin, 2000), Lecture Notes in Physics, pp. 389-427.

5 D. E. Rosner, R. S. Israel, and B. La Mantia, Combustion and Flame 123, 547 (2000).

6 M. Giglio and A. Vendramini, Optics Communications 20, 438 (1977).

7 K. J. Zhang, M. E. Briggs, R. W. Gammon, and J. V. Sengers, J. Chem. Phys. 104, 6881 (1996).

8 S. A. Putnam and D. G. Cahill, Review of Scientific Instruments 75, 2368 (2004).

9 M. M. Bou-Ali, O. Ecenarro, J. A. Madariaga, and C. M. Santamaria, Journal of NonEquilibrium Thermodynamics 24, 228 (1999).

10 M. Giglio and Vendrami.A, Applied Physics Letters 25, 555 (1974).

11 R. Rusconi, L. Isa, and R. Piazza, Journal of the Optical Society of America B-Optical Physics 21, 605 (2004).

12 W. Köhler and R. Schäfer, in New Developments in Polymer Analytics Ii, edited by M. Schmidt (Springer, Berlin, 2000), vol. 151 of Advances in Polymer Science, pp. 1-59.

13 J. C. Bacri, A. Cebers, A. Bourdon, G. Demouchy, B. M. Heegaard, and R. Perzynski, Physical Review Letters 74, 5032 (1995).

14 H. Ning, R. Kita, H. Kriegs, J. Luettmer-Strathmann, and S. Wiegand, J. Phys. Chem. B 110, 10746 (2006).

15 M. E. Schimpf and J. C. Giddings, Journal of Polymer Science Part B-Polymer Physics 27, 1317 (1989).

16 S. Rondot, O. Aaboubi, P. Baudart, D. Erre, E. Merienne, and J. M. Patat, Eur. Phys. J. 17, 
75 (2002).

17 S. Duhr, S. Arduini, and D. Braun, Eur. Phys. J. E 15, 277 (2004).

18 J. K. Platten, M. M. Bou-Ali, P. Costeseque, J. F. Dutrieux, W. Kohler, C. Leppla, S. Wiegand, and G. Wittko, Philos. Mag. 83, 1965 (2003).

19 W. Köhler and B. Müller, J. Chem. Phys. 103, 4367 (1995).

20 M. M. Bou-Ali, O. Ecenarro, J. A. Madariaga, C. M. Santamaria, and J. J. Valencia, J.Phys.:Condens. Matter 10, 3321 (1998).

21 J. F. Dutrieux, J. K. Platten, G. Chavepeyer, and M. M. Bou-Ali, J. Phys. Chem. B 106, 6104 (2002).

22 R. Kita, S. Wiegand, and J. Luettmer Strathmann, J. Chem. Phys. 121, 3874 (2004).

23 P. Kolodner, H. Williams, and C. Moe, J. Chem. Phys. 88, 6512 (1988).

24 W. Köhler and P. Rossmanith, J. Phys. Chem. 99, 5838 (1995).

25 K. B. Haugen and A. Firoozabadi, J. Phys. Chem. B 110, 17678 (2006).

26 P. Polyakov, M. Zhang, F. Müller-Plathe, and S. Wiegand, J. Chem. Phys. 127, 014502 (2007).

27 J. Rauch and W. Köhler, Physical Review Letters 88, art. no. (2002).

28 H. Ning, J. Buitenhuis, J. K. G. Dhont, and S. Wiegand, J. Chem. Phys. 125, 204911 (2006).

29 K. Thyagarajan and P. Lallemand, Optics Communications 26, 54 (1978).

30 D. W. Pohl, Physics Letters A 77, 53 (1980).

31 G. Wittko and W. Kohler, Philos. Mag. 83, 1973 (2003).

32 H. Ning, R. Kita, and S. Wiegand, Progr. Colloid Polym. Sci. 133, 111 (2006).

33 G. M. Hale and M. R. Querry, Appl. Optics 12, 555 (1973).

34 K. F. Palmer and D. Williams, J. Opt. Soc. Am. 64, 1107 (1974).

35 A. Becker, W. Köhler, and B. Müller, Ber. Bunsen-Ges-Phys. Chem. 99, 600 (1995).

36 D. James, J. Mater. Sci 3, 540 (1968).

37 S. Kessler and R. Kowarschik, Opt. Quant. Electron. 7, 1 (1975).

38 M. M. Bou-Ali, O. Ecenarro, J. A. Madariaga, S. C. M., and J. Valencia, Entropie 218/219, 5 (1999).

39 G. Wittko and W. Köhler, Europhys. Lett. 78, 46007 (2007).

40 P. Polyakov and S. Wiegand, J. Chem. Phys. (2007), submitted. 